\title{
Correlações entre força de preensão manual e variáveis antropométricas da mão de jovens adultos
}

\author{
Correlations between grip strength and anthropometric variables of young adults'band
}

Luciane Fernanda Rodrigues Martinho Fernandes ${ }^{1}$, Dernival Bertoncello ${ }^{2}$, Nanci Mendes Pinheiro ${ }^{3}$, Luana Cosenza Drumond ${ }^{4}$

Estudo desenvolvido no Laboratório de Bioengenharia do Curso de Fisioterapia da UNIUBE - Universidade de Uberaba, Uberaba, MG, Brasil

1 Doutora - Professora adjunto - Universidade Federal do Triângulo Mineiro (UFTM) Uberaba (MG), Brasil.

${ }^{2}$ Doutor - Professor adjunto da UFTM - Uberaba (MG), Brasil.

${ }^{3}$ Mestre pela UFTM - Uberaba (MG), Brasil.

${ }^{4}$ Fisioterapeuta graduada pela Universidade de Uberaba (UNIUBE) - Uberaba (MG), Brasil.

ENDEREÇO PARA

\section{CORRESPONDÊNCIA:}

Luciane Fernanda Rodrigues Martinho Fernandes - Avenida Dona Maria de Santana Borges, 1.600, casa 11 Olinda - CEP: 38055-000 Uberaba (MG), Brasil - E-mail: Ifrm@terra.com.br

APRESENTACÃO

maio 2010

ACEITO PARA PUBLICAÇÃO jan. 2011

FONTE DE FINANCIAMENTO: Programa de apoio a pesquisa (PAPE) da Universidade de Uberaba (UNIUBE) e Bolsa de Iniciação Científica PIBICCNPq.

CONFLITO DE INTERESSES: nada a declarar

Apresentação do resumo no $\checkmark$ Seminário de Iniciação Científica - UNIUBE, 2004, Uberaba - MG.
RESUM0: O objetivo do estudo foi correlacionar a força de preensão palmar em diferentes posições de abertura, com variáveis antropométricas da mão. Participaram 73 voluntários de ambos os sexos (40 homens e 33 mulheres), com idade média de 23 $\pm 4,61$ anos. As medidas antropométricas largura da palma, comprimento do dedo indicador, espessura da palma, largura da mão, circunferência da palma e da mão foram realizadas bilateralmente. Para as medidas lineares, utilizou-se um paquímetro digital e, para as medidas de circunferência, a fita métrica. As medidas de força de preensão foram realizadas por meio de um dinamômetro hidráulico (UFTM) Jamar ${ }^{\circledR}$. Os valores antropométricos foram correlacionados com as medidas de força nas cinco posições de abertura. Os homens e as mulheres tiveram maior força na mão direita e, entre as posições, a maior força dos homens foi na de número $3(45,5 \pm 15,53 \mathrm{kgf})$ e das mulheres, na de posição 2 (25,5 $\pm 6,28 \mathrm{kgf})$. Verificaram-se correlações significativas entre a força e os valores antropométricos nas medidas da largura da mão e circunferência da palma - que representam a maior medida transversal e o maior trofismo da mão, respectivamente, no grupo dos homens e a medida de comprimento do dedo, que representa a maior medida longitudinal no grupo das mulheres. Com isso, pode-se concluir que, para os homens, a maior força está relacionada à maior medida transversal e ao maior trofismo da mão e, para as mulheres, ao maior comprimento longitudinal da mão.

Descritores: antropometria; força da mão; dinamômetro de força muscular.

ABSTRACT: The aim of this study was to correlate the grip strength of hand, in different opening positions, with hand anthropometric measures. The study included 73 volunteers (40 male and 33 female) with mean age of $23 \pm 4.61$ years old. Anthropometric measures, such as palm width, forefinger length, palm thickness, hand width, palm and hand circumferences, were performed bilaterally. For linear measures, a digital caliper rule was used as well as a tape measure for circumference measures. Grip strength measures were performed by means of a Jamar ${ }^{\circledR}$ hydraulic dynamometer. Anthropometric values were correlated with strength measures in the five positions of the opening. Both men and women had greater strength in the right hand. Regarding the positions, the bigger strength of men was in the number $3(45.5 \pm 15.53 \mathrm{kgf})$ and that of women in position $2(25.5 \pm 6.28 \mathrm{kgf})$. Significant correlations were verified among strength and anthropometric values in the hand width measure and palm circumference - that represent the greater transversal measure and the greater throphism of left hand, respectively, in men's group -, and the finger length measure, that represents the greater longitudinal measure in women's group. Thus, it may be concluded that for men the greater strength is related to the greater transversal measure and to the greater throphism of hand, while for women, it is related to the greater longitudinal length of hand.

KeYwoRDs: anthropometry; hand strength; muscle strength dynamometer. 


\section{INTRODUCÃO}

A avaliação da força por meio de dinamômetros é uma das mensurações mais fáceis e mais objetivas para a mão. Essas medidas quantitativas passaram a ter grande importância para normatização da força de diferentes populações ${ }^{1,2}$, para diagnóstico de doenças neuromusculares ${ }^{3}$ e para avaliação após procedimentos cirúrgicos ${ }^{4,5}$.

Diversos instrumentos têm sido projetados para a mensuração da força de preensão, desde a simples adaptação de equipamentos para pressão sanguínea ${ }^{6}$, até equipamentos diferenciados confeccionados por An et al. ${ }^{7}$ para medidas da força da mão. Entretanto, um equipamento que ganhou aceitação clínica e tem sido muito utilizado nos estudos da força de preensão é o dinamômetro hidráulico Jamar $^{\circledR 1,2,8-10}$. É constituído de duas barras de aço ligadas, e o sujeito é orientado a tentar aproximá-las. Possui cinco ajustes da posição de abertura para acomodar os diferentes tamanhos de mãos ${ }^{11}$.

$\mathrm{Na}$ ergonomia, um dos problemas encontrados é a dificuldade em se adaplizam a força de preensão aos diferentes tamanhos de mãos. A forma e o tamanho de uma ferramenta têm efeito direto na força de preensão e na sobrecarga biode preensão e o estresse físico resultante nos tendões flexores dos dedos variam de acordo com o tamanho do objeto. Ao segurar uma ferramenta com grande tarem instrumentos de trabalho que utimecânica do membro superior. A força

abertura da mão, as forças do tendão podem ser de duas a três vezes maiores do que uma força aplicada na região mais proximal dos dedos. Por outro lado, se um objeto é muito pequeno, os dedos não podem efetivamente aplicar uma força a ele, porque os músculos flexores estão extremamente encurtados ${ }^{12}$.

A relação da força de preensão com a posição da abertura da mão ou empunhadura tem sido investigada por alguns autores. As medidas da abertura da mão no dinamômetro Jamar ${ }^{\circledR}$ são: posição $1: 3,5 \mathrm{~cm}$; posição $2: 4,8 \mathrm{~cm}$; posição $3: 6,0 \mathrm{~cm}$; posição $4: 4,8 \mathrm{~cm}$ e posição 5:8,6 cm ${ }^{13}$. Em condições mais próximas das encontradas nos ambientes de trabalho, verificou-se que a força de preensão diminui nas aberturas maiores que $5,6 \mathrm{~cm}$ para a preensão ${ }^{14}$ e aumenta na segunda e terceira posições ${ }^{9,15,16}$.

Poucos trabalhos correlacionaram as medidas de força de preensão com medidas antropométricas da mão. No trabalho realizado em meninos praticantes de basquetebol ${ }^{17}$, avaliou-se a influência de medidas antropométricas corpóreas (IMC), medidas de comprimento de dedos e perímetros da mão na força de preensão, encontrando-se correlações significativas entre a força de preensão e medidas antropométricas das mãos. Ruiz-Ruiz et al. ${ }^{13}$ mensuraram o tamanho da mão, como a medida entre a extremidade do polegar e a extremidade do quinto dedo na região palmar, com os dedos em abdução total e correlacionaram essa medida com a medida de força máxima.

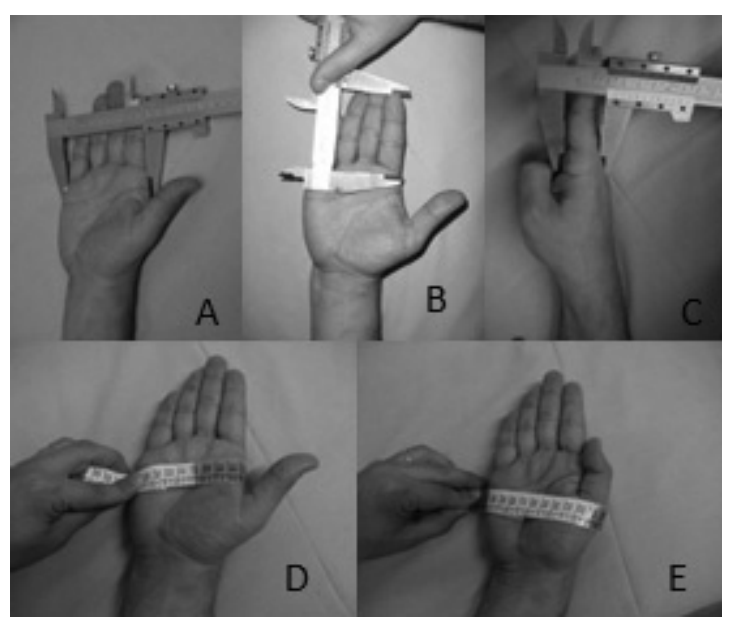

Figura 1. Medidas antropométricas da mão. (A) largura da palma; (B) comprimento do dedo; (C): espessura da palma; (D): circunferência da palma; (E): circunferência da mão.
Os autores encontraram uma associação entre a força e o tamanho da mão nas mulheres. Firrell e Crain ${ }^{15}$ correlacionaram a medida da força máxima com quatro medidas da mão e encontraram correlação entre o comprimento do dedo e a posição máxima do dinamômetro. Portanto, o objetivo deste trabalho foi correlacionar a força de preensão palmar nas diferentes posições de abertura da mão com variáveis antropométricas manuais buscando contribuir para a confecção e/ou adaptação de ferramentas de trabalho manual.

\section{MÉTODOS}

\section{Casuística}

Participaram deste estudo 73 voluntários de ambos os sexos (40 homens e 33 mulheres), com idade média de 23 $\pm 4,61$ anos. Os critérios de inclusão foram: a) ausência de doenças neuromusculares e musculoesquelética nos membros superiores; b) sedentários; c) idade entre 18 e 39 anos.

Todos os voluntários foram informados sobre os objetivos e procedimentos da pesquisa e assinaram um termo de consentimento livre e esclarecido, conforme resolução 196/96 do Conselho Nacional de Saúde (CNS). A pesquisa foi aprovada pelo Comitê de Ética em Pesquisa da Universidade de Uberaba, com parecer PRO-CEP 012/03.

\section{Procedimentos}

Realizaram-se medidas antropométricas da mão e medidas de força isométrica máxima de preensão palmar. As medidas antropométricas foram executadas bilateralmente, com o voluntário sentado e a mão apoiada sobre uma mesa. Essas medidas foram: largura da palma (LP); comprimento do dedo indicador (CD); espessura da palma (EP); largura da mão (LM); circunferência da palma (CP); e circunferência da mão $(\mathrm{CM})^{12}$ (Figura 1 ). Para as medidas antropométricas lineares, utilizou-se um paquímetro e, para as medidas de circunferência, utilizou-se uma fita métrica.

As medidas de força de preensão foram realizadas por meio de um dinamômetro hidráulico da marca Jamar ${ }^{\circledR}$, com graduação de 0 a 90 kgf. Utilizaram-se 
as cinco posições do dinamômetro. A posição para a realização do teste foi com o indivíduo sentado, o ombro aduzido e em rotação neutra, o cotovelo fletido a $90^{\circ}$ e o antebraço e punho em posição neutra $^{18}$.

Após treinamento prévio, realizou-se apenas uma medida para cada abertura da mão, a fim de se verificar a contração voluntária máxima em uma repetição, alternando as mãos em cada posição para evitar a fadiga, sempre começando da posição 1 (menor abertura da mão) até a posição 5 (maior abertura da mão) do dinamômetro. Os voluntários foram orientados a manter a contração por três segundos e, ao final das mensurações, realizaram alongamentos para os músculos do punho e dedos.

Previamente à coleta da força, o dinamômetro foi calibrado com massas de chumbo, e o coeficiente de correlação linear foi de 0,997.

\section{Análise estatística}

Os dados foram submetidos a análise estatística descritiva e inferencial, com nível de significância de 5\%. Na análise estatística descritiva, utilizou-se a média e o desvio padrão. Na estatística inferencial, para a verificação da normalidade e da homocedasticidade, utilizaram-se os testes Kolmogorov-Smirnov (K-S) e Bartlett, respectivamente, e, posteriormente, a ANOVA para comparação das medidas antropométricas e medidas de força entre os homens e mulheres, nas diferentes posições. Para os dados em que a ANOVA foi significativa, utilizou-se o Teste de Tukey.

No estudo da correlação entre as medidas antropométricas e a forças de preensão palmar nas diferentes posições, utilizou-se o coeficiente de correlação (r) de Pearson. A significância do coeficiente de correlação foi baseada na distribuição de Student. Os dados foram processados por meio do software Statistica (versão 5.1).

\section{RESULTADOS}

Os voluntários foram divididos em dois grupos: homens e mulheres. Em relação às características da amostra, observam-se diferenças estatisticamente significativas para os valores da massa corporal e estatura entre os grupos (Tabela 1). O lado esquerdo foi o dominante em 4 (10\%) homens e em $2(6,1 \%)$ mulheres.

Quanto à antropometria da mão, em todas as variáveis os valores médios dos homens foram maiores que os das muIheres, tanto na mão direita quanto na esquerda (Figura 2).

Os valores antropométricos apresentaram distribuição normal e homocedasticidade. Encontraram-se diferenças significativas para todas as medidas, tanto do lado direito quanto do esquerdo entre

os grupos $(p<0,05)$. Em relação ao lado, observaram-se valores maiores nas medidas das mãos para o membro superior direito, apesar de essa diferença não ser significativa $(p>0,05)$.

Em relação à força de preensão palmar, verificaram-se maiores valores da força na mão direita, para ambos os grupos, com exceção da posição 1 do dinamômetro para os homens. O maior valor da força nos homens foi na posição $3(45,5 \pm 15,53$ kgf) e, nas mulheres, na posição 2 (25,5 $\pm 6,28$ kgf), ambos na mão direita (Figura 3).

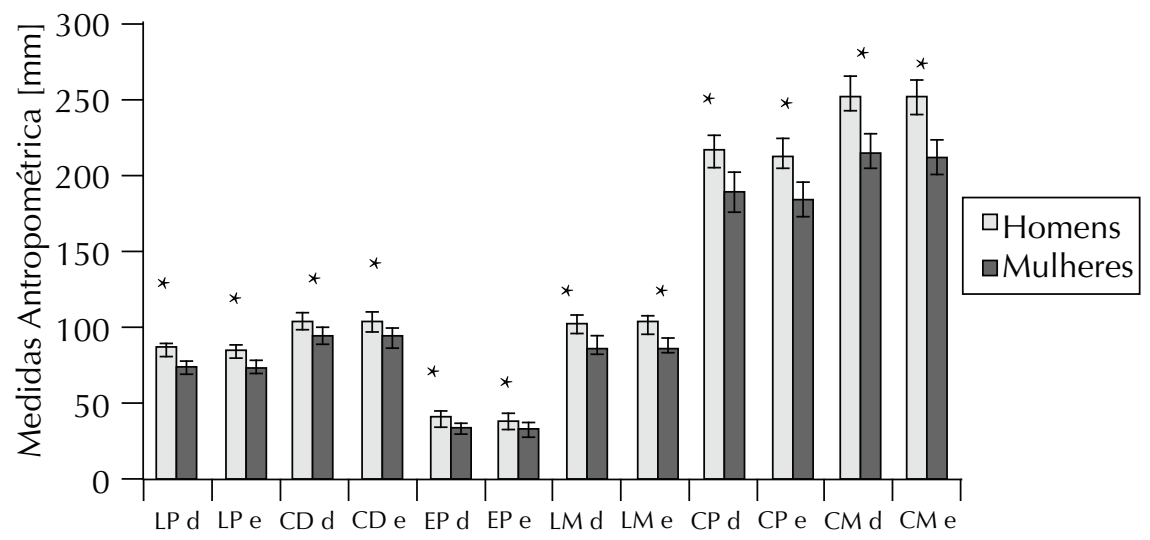

Figura 2. Valores da média e desvio padrão das variáveis antropométricas das mãos direita e esquerda entre os homens e as mulheres.

LP: largura da palma; CD: comprimento do dedo indicador; EP: espessura da palma; LM: largura da mão; CP: circunferência da palma; CM: circunferência da mão; d: direito; e: esquerdo. Diferenças significativas para todas as variáveis tanto do lado direito quanto do esquerdo entre homens e mulheres $(p<0,0001)$.

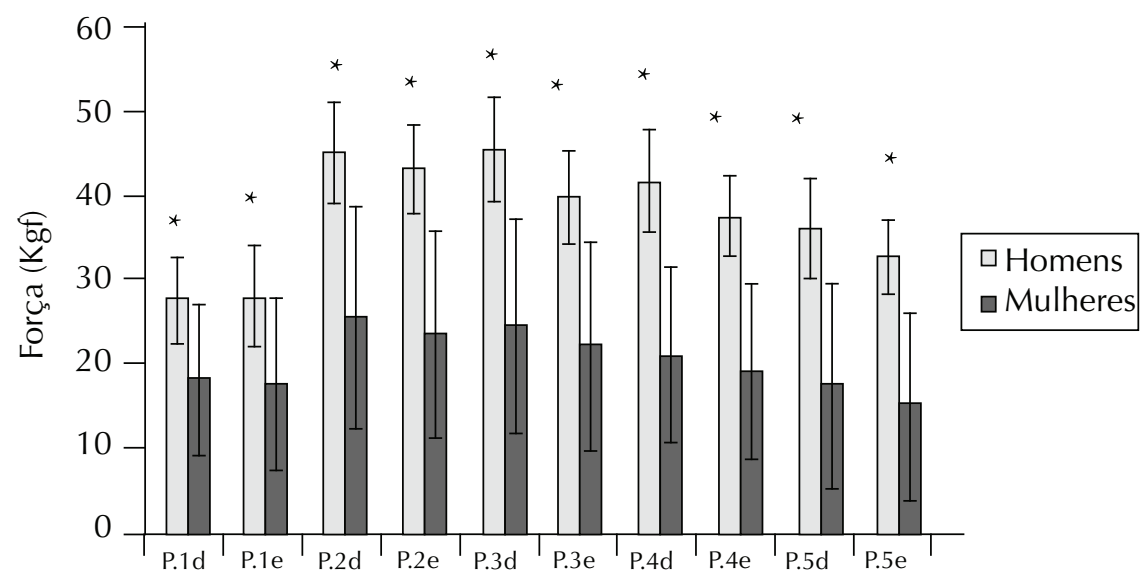

Figura 3. Valores da média e desvio padrão da força de preensão palmar (kgf), nas diferentes posições do dinamômetro, para as mãos direita e esquerda dos homens e mulheres.

P: força de preensão; 1 a 5: posições de abertura da mão; d: mão direita; e: mão esquerda. Diferença significativa para todas as posições do dinamômetro tanto para o lado direito quanto para o lado esquerdo entre homens e mulheres $(p<0,0001)$. 
Os valores da força de preensão palmar nas diferentes posições apresentaram distribuição normal e homocedasticidade. Realizou-se a ANOVA para se verificarem diferenças entre homens e mulheres nas diferentes posições (sexo versus posição). Como a ANOVA foi significativa, realizou-se o Teste de Tukey. Na comparação entre os homens e muIheres, a diferença foi significativa para todas as posições $(p<0,05)$.

Calcularam-se as correlações entre as seis medidas antropométricas e as cinco medidas de força, para o lado direito e para o lado esquerdo, tanto para os homens quanto para as mulheres. Os valores das correlações estão apresentados na Tabela 2.

Em se tratando das correlações, para o grupo dos homens, foram encontradas correlações significativas, na mão direita, para LM e CP nas cinco posições; para LP, nas posições 2, 3, 4 e 5; para CD e CM, nas posições 4 e 5 . Para a mão esquerda, as correlações foram significativas para LP e CD na posição 4; para EP, LM, CP e CM, nas posições 2, 4 e 5 .
No grupo das mulheres, foram poucas as correlações significativas na mão direita e apresentaram-se da seguinte forma: LP nas posições 4 e 5; CD nas posições 3, 4 e 5. Para a mão esquerda, as correlações foram significativas para LP nas posições 4 e 5 ; CD nas posições 2, 3, 4 e 5; EP na posição 4; LM e CM nas posições 3,4 e 5 .

\section{DISCUSSÃO}

A escolha das seis variáveis antropométricas foi devida a três fatores: 1) facilidade na delimitação e na realização das medidas; 2) as seis medidas são representativas para a antropometria da mão; 3) sua utilização na ergonomia para confecção de equipamentos de proteção individual.

Em relação ao número de medidas antropométricas para a mão, foram encontrados na literatura trabalhos com $1^{13,16}, 4^{15}, 15^{17}, 20^{19}$ e 24 medidas $^{20}$. As seis medidas escolhidas para este estudo são representativas para a antropometria

Tabela 1. Caracterização da amostra (média \pm desvio padrão)

\begin{tabular}{lccc}
\hline & $\begin{array}{c}\text { Massa corporal } \\
(\mathrm{kg})\end{array}$ & $\begin{array}{c}\text { Estatura } \\
(\mathrm{m})\end{array}$ & $\begin{array}{c}\text { Idade } \\
(\text { anos })\end{array}$ \\
\hline Homens $(\mathrm{n}=40)$ & $76,69 \pm 11,05^{*}$ & $1,78 \pm 0,07^{* *}$ & $22,8 \pm 4,72$ \\
Mulheres $(\mathrm{n}=33)$ & $57,3 \pm 10,29^{*}$ & $1,60 \pm 0,06^{* *}$ & $22,9 \pm 4,54$ \\
\hline
\end{tabular}

${ }^{*} \mathrm{p}<0,00000 ;{ }^{* *} \mathrm{p}<0,00000$. Teste $t$ independente para grupos. da mão, pois abrangem comprimento, largura e espessura.

Neste estudo, para as variáveis antropométricas, os valores médios dos homens foram significativamente maiores para todas as medidas, o que se relaciona ao fato de também apresentarem diferenças significativas da massa corporal e estatura. Quando comparados os dados obtidos com os de Webb (1978) apud Chaffin et al. ${ }^{12}$, os valores das medidas antropométricas da mão foram semeIhantes, com exceção da medida do comprimento do dedo. Wagner ${ }^{19}$ realizou um trabalho sobre a antropometria e a amplitude de movimento das mãos de pianistas para contribuir na ergonomia de instrumentos musicais. Realizou 20 medidas de dimensão do tamanho da mão e de todas essas medidas. Somente duas são comparáveis às medidas realizadas no presente trabalho. A medida 2 de Wagner corresponde à LP e a medida 9 corresponde à $\mathrm{CD}$. Os valores da medida da LP foram semelhantes nos dois estudos, porém o mesmo não ocorreu para a medida do CD. Essa medida foi a mais difícil de ser obtida, devido à dificuldade em alinhar o paquímetro na articulação metacarpofalangeana do segundo dedo.

Na medida da força de preensão palmar, verificaram-se valores significativamente maiores nas medidas de força dos homens e no membro superior direito,

Tabela 2. Valores das correlações entre as medidas antropométricas e medidas de força de preensão palmar

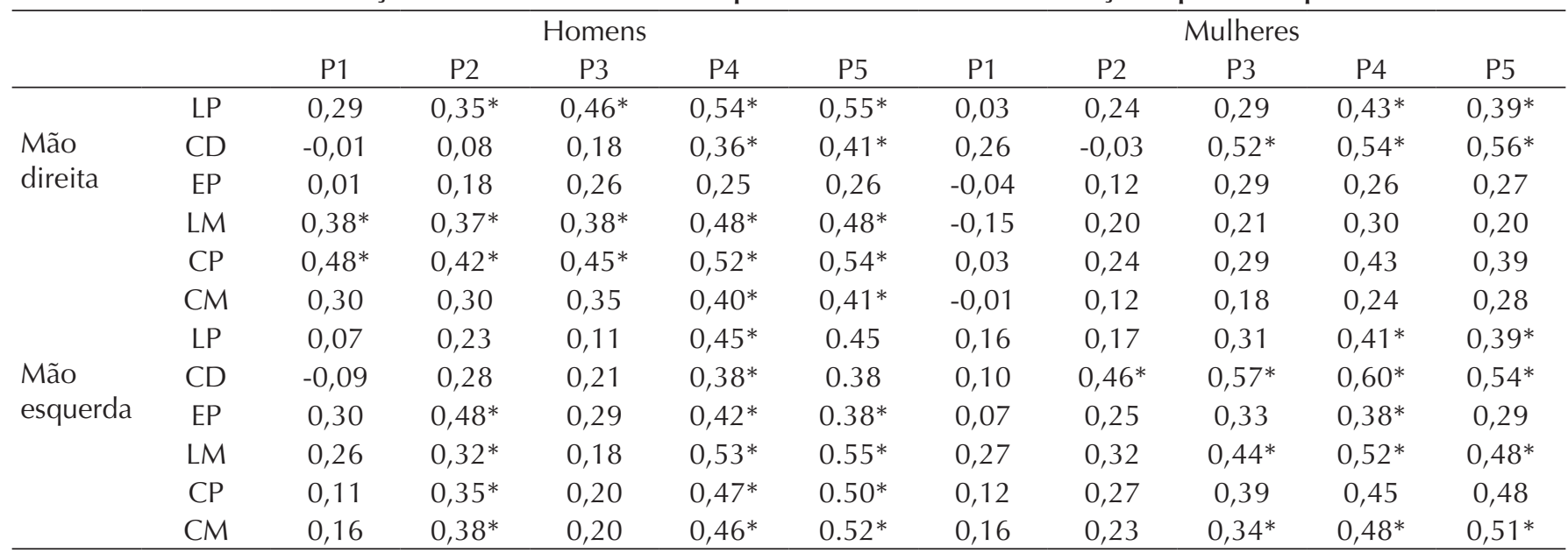

LP: largura da palma; CD: comprimento do dedo indicador; EP: espessura da palma; LM: largura da mão; CP: circunferência da palma; CM: circunferência da mão .P1-P5: posição.

*significativo para $\mathrm{p}<0,05$. 
exceto na posição 1 do dinamômetro para os homens, que apresentou maior força na mão esquerda. Não foi possível comparar os dados - pois outros autores realizaram apenas a medida na posição 2 do dinamômetro - e foram divididos em lado dominante e não dominante ${ }^{1,2}$.

Quando analisada a medida da força em relação à posição de abertura da mão, a maior força dos homens foi na posição 3 e, para as mulheres, na posição 2 , ambos do lado direito. No estudo realizado, o tamanho da mão dos homens foi significativamente maior e acredita-se que isso refletiu na maior força na posição 3 . Os resultados deste estudo corroboram outros anteriores ${ }^{9,15,16}$, que investigaram a diferença na força de preensão nas cinco posições de abertura do dinamômetro e observaram que as maiores forças encontradas foram também na segunda e terceira posições.

Segundo Chaffin ${ }^{12}$, a maior força de preensão ocorre quando a ferramenta pode ser totalmente envolvida pela mão, sendo que a força não deve ser aplicada na região mais distal dos dedos nem na região mais proximal. Além disso, Imrhan ${ }^{14}$ salienta a importância de aplicação das medidas de força de preensão associadas ou não à avaliação de desempenho de tarefas, como um cuidado que deve ser exercido em função da variação da força nas diferentes aberturas dos equipamentos.

A correlação entre as medidas da mão do usuário e a força necessária para a atividade torna-se importante para prevenir o dispêndio de força. Portanto, é importante que o indivíduo utilize uma ferramenta com tamanho adequado para a sua mão, a fim de que seja somente aplicada força necessária para a realização de uma atividade como a de precisão, por exemplo.

A posição 2 do dinamômetro Jamar ${ }^{\circledR}$ é considerada a mais eficiente para testes de força e tem sido adotada pela American Society of Hand Therapists para testes rotineiros ${ }^{18}$. Entretanto, no presente estudo, como no de Härkönen et $a /^{16}$, a posição 3 foi a mais eficiente para os homens e a posição 2 para as mulheres. Por consequência, não deve ser adotada uma única posição para o teste sem considerar o tamanho da mão, já que os dinamômetros foram confeccionados com diferentes posições de abertura para adaptação aos diferentes tamanhos de mãos. Oh e Radwin ${ }^{21}$ sugerem que, para mãos pequenas e médias, a força máxima de preensão acontece em aberturas próximas de $5 \mathrm{~cm}$ (posição 2 do dinamômetro); para mãos maiores, ela fica em torno de $6 \mathrm{~cm}$ (posição $3 \mathrm{do}$ dinamômetro).

A correlação entre medidas antropométricas da mão e medida de força da preensão palmar foi avaliada por Firrelll and Crain ${ }^{15}$, que realizaram quatro medidas antropométricas da mão e correlacionaram com medida de força de preensão máxima. Os autores encontraram somente correlação entre o comprimento dos dedos e a posição máxima do dinamômetro.
Em relação às correlações entre as variáveis antropométricas e dinamométricas, os homens apresentaram um número maior de correlações significativas que as mulheres. A medida LM está relacionada à maior medida transversal e a $\mathrm{CP}$, ao trofismo da mão. Para as mulheres, as correlações mais significativas foram em relação ao $\mathrm{CD}$, que representa a maior medida linear de direção longitudinal.

Os resultados deste estudo fornecem informações de que a relação entre a força de preensão e antropometria da mão difere entre os homens e mulheres, e esses dados podem ser utilizados para projetar ferramentas e equipamentos de manuseio ou de proteção individual de maneiras diferentes para os sexos. Com os resultados apresentados, espera-se uma maior preocupação na confecção de ferramentas com adaptações especificas para homens e mulheres e para cada atividade, buscando-se uma maior eficiência e menor sobrecarga dos músculos da mão.

\section{CONCLUSÕES}

Conclui-se que a correlação significativa da força de preensão está relacionada à maior medida transversal (largura da mão) e ao maior trofismo da mão (circunferência da mão) para os homens e ao maior comprimento longitudinal da mão, que corresponde à medida de comprimento do dedo, para as mulheres.

\section{REFERÊNCIAS}

1. Mathiowetz V, Kashman N, Volland G, Webwer K, Dowe M, Rogers S. Grip and pinch strenght: normative data for adults. Arch Phys Med Rehabil. 1985;66(2):6974 .

2. Caporrino FA, Faloppa F, Santos JBG, Réssio C, Soares FHC, Nakachima LR, et al. Estudo populacional da força de preensão palmar com dinamômetro Jamar ${ }^{\circledR}$. Rev Bras Ortop. 1998;33(2):150-4.

3. Dellon AL, Keller KM. Computer-assisted quantitative sensoriomotor testing in patients with carpal and cubital tunnel syndromes. Ann Plast Surg. 1997;38(5):493-502.

4. Fuss FK, Wagner TF. Biomechanical alterations in the carpal arch and hand muscles after carpal tunnel release: a further approach toward understanding the function of the flexor retinaculum and the cause of postoperative grip weakness. Clin Anat. 1996;9(2):100-8.

5. Netscher D, Mosharrafa A, Lee M, Polsen C, Choi H, Steadman AK, et al. Tranverse carpal ligament: its effect on flexor tendon excursion, morphologic changes of the carpal canal, and on pinch and grip strengths after open carpal release. Plast Reconstr Surg. 1997;100(3):636-42.

6. Kowanko ICR, Knapp MS, Pownall R, Swannell AJ. Domiciliary self-measurement in rheumatoid arthritis and the demonstration of circadian rhythmicity. Ann Rheum Dis. 1982;41(5):453-5. 


\section{Referências (cont.)}

7. An K-N, Chao EYS, Askey LJ. Hand strength measurements intruments. Arch Phys Med Rehabil. 1980;61:366-8.

8. Mathiowetz V, Wiemer DM, Federman SM. Grip and pinch strength: norms for 6- to 19-years-olds. Am J Occup Ther. 1986;40(10):705-11.

9. Goldman S, Calahan TD, An KN. The injured upper extremity and the JAMAR five-handle position grip test. Am J Phys Med Rehabil. 1991;70(6):306-8.

10. Bellace JV, Healy D, Besser MP, Byron T, Hohman L. Validity of the Dexter Evaluation System's Jamar dynamometer attachment for assessment of hand grip strength in a normal population. J Hand Ther. 2000;13(1):46-51.

11. Blair V. Função da mão. In: Durward BR, Baer GD, Rowe PJ. Movimento funcional humano: mensuração e análise. São Paulo: Manole; 2001. p. 159-79.

12. Chaffin DB, Andersson GBJ, Martin BJ. Occupational Biomechanics. $3^{\text {rd }}$ ed. New York: Wiley-Interscience; 1999.

13. Ruiz-Ruiz J, Mesa JL, Gutiérrez A, Castilho MJ. Hand size influences optimal grip span in woman but not in men. J Hand Surg Am. 2002;27(5):897-901.
14. Imrhan SN, Rahman R. The effects of pinch width on pinch strengths of adult males using realistic pinchhandle coup ling. Int J Ind Ergon. 1995;16(2):123-34.

15. Firrell JC, Crain GM. Which setting of the dynamometer provides maximal grip strength? J Hand Surg Am. 1996;21(3):397-401.

16. Härkönen R, Piirtomaa M, Alaranta H. Grip strength and hand position of the dynamometer in 204 Finnish adults. J Hand Surg Br. 1993;18(1):129-32.

17. Visnapuu M, Jürimäe T. Handgrip strength and hand dimensions in young handball and basketball players. J Strength Cond Res. 2007;21(3):923-9.

18. Balogun JA, Akomolafe CT, Amusa LO. Grip strength: effects of testing posture and elbow position. Arch Phys Med Rehabil. 1991;72(5):280-3.

19. Wagner $\mathrm{CH}$. The pianist's hand: anthropometry and biomechanics. Ergonomics. 1988;31(1):97-131.

20. Imrhan SN, Sarder MD, Mandahawi N. Hand anthropometry in Bangladeshis living in America and comparisons with other populations. Ergonomics. 2009;52(8):987-98.

21. Oh S, Radwin RG. Pistol grip power tool handle and trigger size effects on grip exertions and operator preference. Hum Factors. 1993;35(3):551-69. 